\title{
Emittance compensation of compact superconducting guns and booster linac system
}

\author{
Xiangyun Chang, Ilan Ben-Zvi, and Jörg Kewisch \\ Brookhaven National Laboratory, Upton, New York 11973, USA \\ (Received 30 September 2005; revised manuscript received 5 January 2006; published 14 April 2006)

\begin{abstract}
We discuss the mechanics of emittance compensation for a compact superconducting rf gun with a solenoid, a drift space, and a linac system, and give general rules for designing such a system. It is preferable to ensure that the cathode is recessed and that the solenoid is close to it. The drift length, the solenoid's strength, the size of the waist spot, and the linac's accelerating gradient can be determined from the size of the beam's spot at the gun exit, all of which are easily obtained from simulations. Chromaticity effects generally start at the solenoid but can be ignored in the gun. The energy spread extends the spread of the slices' waist positions, and therefore degrades the emittance compensation. The gun cavity should be designed to minimize energy spread at the gun's exit; this can be achieved by shortening the effective length of the cavity cell. The linac gradient required to minimize chromaticity by focusing at the linac entrance is larger than the gradient required for matching the linac's invariant envelope. The applied solution must be a compromise between these two parameters. For a beam with limited energy spread, its waist must be in front of the linac's entrance.
\end{abstract}

DOI: 10.1103/PhysRevSTAB.9.044201

PACS numbers: $29.27 .-\mathrm{a}$

\section{INTRODUCTION}

The mechanics of emittance compensation for a compact rf gun with a solenoid, a drift space, and a linac system was analyzed in detail by Rosenzweig, and Serafini [1] for the case of normal-conducting cavities where the solenoid field is inside the cavity.

For a superconducting cavity the solenoid field can not penetrate the cavity and therefore the emittance compensation has new characteristics. In this paper we describe the beam transport of intense bunches through a compact superconducting gun, followed by a solenoid which is relatively far away from the cathode, a drift space, and booster linac. We describe the process of emittance compensation for such a system, including an analysis of the effect due to chromaticity.

In Sec. II, we review the S-R theory of beam propagation in a constant accelerating structure, and the matching of its invariant envelope. In Sec. III, we extend the S-R invariantenvelope theory to a superconducting rf gun and offer directions on how to choose the system's parameters. Section IV describes our study of the beam's properties in a drift space and gives the conditions for optimizing emittance compensation. The role of emittance compensation for the whole system is summarized in Sec. V. The effect of chromaticity is presented in Sec. VI. Section VII gives a numerical simulation based on the Relativistic Heavy Ion Collider (RHIC) electron-cooling project. Section VIII summarizes the analysis presented in this paper.

\section{THE INVARIANT ENVELOPE}

This section restates the analytical theory of emittance compensation developed by S-R in Ref. [1] that is the starting point for this paper.
Although the real accelerating gradient of an accelerator varies along $z$, the motion can be approximated using a constant (average) acceleration [2]. The small oscillation of the envelope about the constant acceleration envelope can be ignored.

For a $\pi$-mode standing wave linac with a constant average accelerating gradient $\gamma^{\prime}$, the envelope equation has a special solution:

$$
\hat{\sigma}=\left(2 / \gamma^{\prime}\right) \sqrt{I / 3 I_{0} \gamma}, \quad \hat{\sigma}^{\prime}=-\sqrt{I / 3 I_{0} \gamma^{3}} .
$$

$I_{0}=17045 A$ is the Alfvén current, and $I(\zeta)=q \lambda(\zeta) \nu_{b}$ is the local current of a longitudinal slice. $\zeta=z-z_{0}$ is the relative coordinate of the longitudinal bunches' positions, where $z_{0}$ is the center of the bunch, $q$ is the electron charge, $\lambda(\zeta)$ is the local charge line density, $\nu_{b} \approx c$ is the velocity of the beam, and $c$ is the velocity of light.

The solution $\hat{\sigma}$ is called the invariant envelope, and represents the equilibrium state between the focusing force of the average rf acceleration, and the defocusing spacecharge force. The invariant envelopes of the other types of beam transport and acceleration are also given in Ref. [1].

Matching the beam's envelope to the invariant envelope is the preferred mode of propagating the beam, thereby optimizing emittance compensation. To match the invariant envelope of an actual $\pi$-mode linac with a finite length (as schematically shown in Fig. 1), the effect of the focusing effect at the linac's entrance and the defocusing effect at its exit must be included.

The beam experiences a focusing kick at the entrance [2] with $\theta_{i}=\sigma_{i}^{\prime} / \sigma_{i}=-\gamma^{\prime} / 2 \gamma_{i}$ which is equal to $\hat{\sigma}^{\prime} / \hat{\sigma}$; this requires matching the beam's initial size at the linac entrance to that of the invariant envelope,

$$
\sigma_{i}=\left(2 / \gamma^{\prime}\right) \sqrt{I / 3 I_{0} \gamma_{i}}
$$




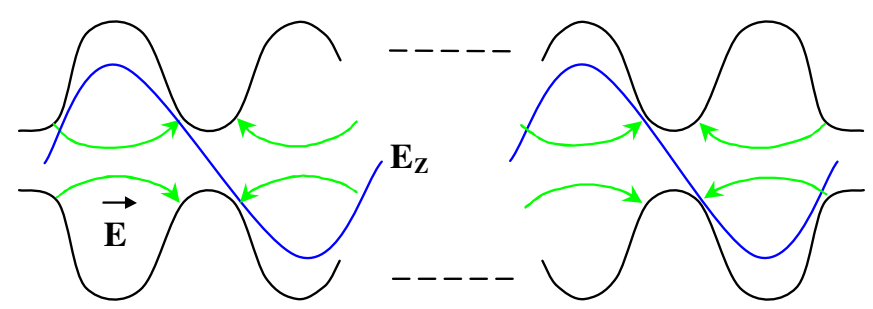

FIG. 1. (Color) Scheme of an $n$-cell $\pi$-mode linac. The green lines represent the field lines while the blue line represents the field strength on the axis.

and a vanishing divergence. At the exit, the beam gets a defocusing kick with $\theta_{f}=\sigma_{f}^{\prime} / \sigma_{f}=\gamma^{\prime} / 2 \gamma_{f}$. Thus, the size of the beam emerging from the linac is

$$
\sigma_{f}=\left(2 / \gamma^{\prime}\right) \sqrt{I / 3 I_{0} \gamma_{f}}
$$

also with vanishing divergence.

\section{MATCHING OF THE SUPERCONDUCTING RF GUN}

One can apply the invariant envelope concept to a rf gun. Consider the multicell rf gun structure shown in Fig. 2.

With the approximation that the beam is relativistic at the cathode (it becomes relativistic in a very short distance thereafter because of the high rf field), the rf kick in the first half-cell is given by [3]:

$$
\theta_{0}=\sigma^{\prime} / \sigma=\gamma^{\prime} / \gamma
$$

To match the invariant envelope of its average acceleration, the beam should have an initial convergence angle of $\theta_{0} \approx-\gamma^{\prime} / \gamma$ and an initial size according to Eq. (2.2). Given that the beam starts out as nonrelativistic, the rf's defocusing strength and the required initial converging angle are even larger. As the beam leaves a flat cathode the electrons move parallel to the axis.

In a normal-conducting gun a strong focusing solenoid field (bucking coil) close to cathode is necessary to avoid too much deviation from the invariant envelope. This is the case analyzed by Rosenzweig and Serafini.

Lengthening the first half-cell reduces the rf's defocusing strength there, so lessening the initial mismatch.

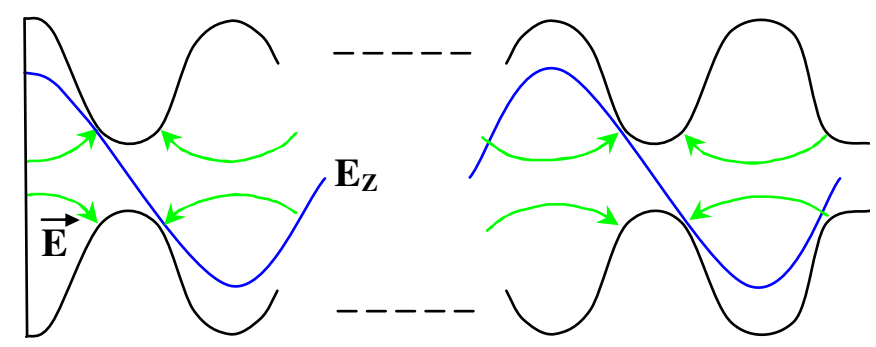

FIG. 2. (Color) A multicell rf gun. The green lines are the electric field lines, and the blue line is the field's strength on the axis.
However, an alternate and more effective way to reduce the initial mismatch is to recess the cathode $[4,5]$ so that the effective rf defocusing in the first half-cell is lowered. Recessing it reduces the required solenoid strength and/ or sacrifices the strength of the electric field on the cathode.

Since it is impossible to obtain focusing by an external solenoid in a superconducting gun, recessing the cathode is the only way to approach matching the invariant envelope. As mentioned in Sec. II, for such matching a parallel beam is necessary at the exit from the acceleration region. Thus, ideally, the recess should generate a parallel beam at the gun's exit. From simulation it is found that the amount of the recess is such that the maximum field on cathode is roughly half of the maximum field on axis.

The recess of the cathode in the superconducting cavity provides the focusing that a solenoid does in a normalconducting cavity. So the optimized spot size $\sigma_{c}$ on cathode is not far from what is determined by the S-R:

$$
\sigma_{c}=\sqrt[3]{(c / 2 \pi) Q A / \Lambda \gamma^{\prime 2}}
$$

where $Q$ is the charge of the bunch, $A$ is the aspect ratio of the transverse spot size and longitudinal bunch length. It is generally chosen to be about $0.5, \Lambda^{\mathrm{opt}} \equiv I /\left(\gamma^{\prime} \sigma_{c}\right)^{2}$ is the optimized Cauchy current which can be approximated as

$$
\begin{aligned}
\Lambda^{\mathrm{opt}}[k A]= & 57.3-12.4 \alpha+2.63 \alpha^{2}+26.2 A-1.78 \alpha A \\
& +1.86 A^{2},
\end{aligned}
$$

where $\alpha=e E_{0} / 2 k m c^{2}$ represents the dimensionless amplitude of the accelerating field associated with the rf wave number $k$.

\section{OPTIMIZATION OF THE DRIFT SPACE LENGTH}

The drift space length is dependent on the beam conditions at the solenoid which is easy to find by a tracking code. In a drift space, the following is the generalized envelope equation:

$$
\nu^{\prime \prime}(z)-1 / \nu(z)=0 .
$$

$\nu(z) \equiv \sigma(z) / \sqrt{P}$ is the generalized beam size. In this section, "beam size" signifies this generalized beam size. $P=I / 2 I_{0} \gamma^{3}=Q c / 2 \sqrt{2} \pi I_{0} \gamma^{3} \sigma_{z}$ is the normalized, relativistic perveance where $\sigma_{z}$ is the rms bunch length. The longitudinal charge distribution is assumed to be Gaussian.

The integral form of Eq. (4.1) is

$$
\int_{1}^{\nu / \nu_{i}} \frac{d x}{\sqrt{\nu_{i}^{\prime 2}+2 \ln x}}=\frac{z-z_{i}}{\nu_{i}},
$$

where the subscript $i$ stands for the initial position, which is the beginning of the drift space. Equations (4.1) and (4.2) are exactly the same as those in Ref. [1].

Although there is no analytical solution of Eq. (4.2), the envelope can be approximated near the beam's waist [6] by 
a quadratic function:

$$
\nu=\nu_{W}+\frac{\left(z-z_{W}\right)^{2}}{\nu_{W}},
$$

where $z_{W}$ is the position of the beam's waist and $\nu_{W}$ is the generalized beam size at its waist.

The divergence of a beam near the waist then is

$$
\nu^{\prime}=\frac{d \nu}{d z}=\frac{2\left(z-z_{W}\right)}{\nu_{W}} .
$$

The relative position of the beam's waist can be expressed as

$$
Z_{W}\left(\nu_{i}, \nu_{i}^{\prime}\right)=\nu_{i} \int_{e^{-\nu_{i}^{\prime 2} / 2}}^{1} \frac{d x}{\sqrt{\nu_{i}^{\prime 2}+2 \ln x}} .
$$

The integral can be approximated (within a $1 \%$ error) by a function:

$$
\begin{aligned}
g\left(\nu_{i}^{\prime}\right) \equiv & \int_{e^{-\nu_{i}^{\prime 2} / 2}}^{1} \frac{d x}{\sqrt{\nu_{i}^{\prime 2}+2 \ln x}} \\
\approx & 0.0049487 \nu_{i}^{\prime 6}-0.069182 \nu_{i}^{\prime 5}+0.35548 \nu_{i}^{4} \\
& -0.74171 \nu_{i}^{\prime 3}+0.21824 \nu_{i}^{\prime 2}+0.95449 \nu_{i}^{\prime} \\
& +0.0019165
\end{aligned}
$$

in the range $\nu_{i}^{\prime} \leq 4$. The beam's spot size at the waist is

$$
\nu_{W}=\nu_{i} e^{-\nu_{i}^{\prime 2} / 2} .
$$

In general, when the beam enters the drift space from a compact rf gun and solenoid, the multiple-slice phasespace can be schematically shown as in Fig. 3.

From Eq. (4.6) we can estimate the spread of $z_{W}$ caused by a small initial convergence angle spread $\Delta \nu_{i}^{\prime}$

$$
\begin{aligned}
\Delta z_{W}= & \Delta Z_{W} \approx \frac{d Z_{W}}{d \nu_{i}^{\prime}} \Delta \nu_{i}^{\prime} \\
\approx & \nu_{i}\left(0.0294 \nu_{i}^{\prime 5}-0.346 \nu_{i}^{\prime 4}+1.422 \nu_{i}^{\prime 3}-2.225 \nu_{i}^{\prime 2}\right. \\
& \left.+0.4364 \nu_{i}^{\prime}+0.9545\right) \Delta \nu_{i}^{\prime} .
\end{aligned}
$$

The angle spread near the beam's waist, $\Delta \nu_{W}^{\prime}$, can be

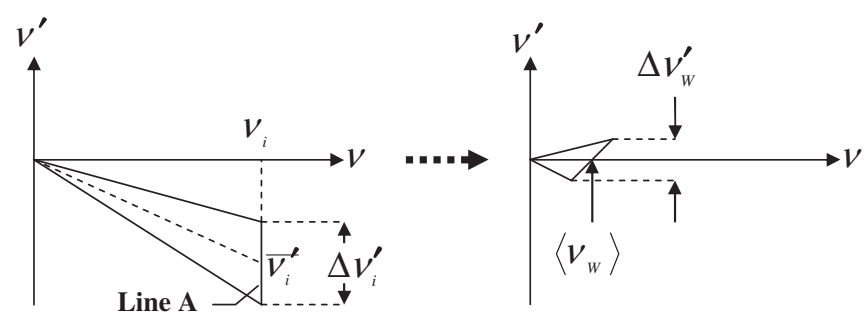

FIG. 3. Left: The phase-space immediately after the solenoid, in which the end points of the slices' emittances are oriented perpendicular to the $\nu$ axis (line A). Right: The phase-space near the beam's waist. estimated from Eq. (4.4):

$$
\Delta \nu_{W}^{\prime} \approx \frac{2 \Delta z_{W}}{\left\langle\nu_{W}\right\rangle} .
$$

Then, the emittance at the average beam waist $\varepsilon_{W}$ in $\left(\nu, \nu^{\prime}\right)$ phase-space is

$$
\varepsilon_{W} \approx \frac{1}{2} \Delta \nu_{W}^{\prime}\left\langle\nu_{W}\right\rangle \approx \Delta z_{W} .
$$

Figure 4 plots the normalized emittance at the beam's waist as a function of the average initial convergence angle $\bar{\nu}_{i}^{\prime}$.

We see that to attain the minimum emittance at the beam's waist, the optimized average initial convergence angle is $\left|\bar{\nu}_{i}^{\prime}\right| \approx 1$.3. In configuration space, the corresponding initial angle, the position of the beam's waist, and its spot size are, respectively,

$$
\begin{aligned}
\left|\sigma_{i}^{\prime}\right| & =1.3 \sqrt{P} \approx 0.0244 \frac{\sqrt{Q[\mathrm{nC}]}}{\sqrt{\sigma_{z}[\mathrm{~cm}] \gamma^{3 / 2}}} \\
Z_{W}[\mathrm{~cm}] & =0.765 \nu_{i} \approx 40.6 \frac{\sigma_{i}[\mathrm{~cm}] \sqrt{\sigma_{z}[\mathrm{~cm}]} \gamma^{3 / 2}}{\sqrt{Q[\mathrm{nC}]}}, \\
\sigma_{W} & =\sigma_{i} e^{-\nu_{i}^{\prime 2} / 2} \approx 0.43 \sigma_{i} .
\end{aligned}
$$

Equations (4.11a)-(4.11c) are the relations between the optimized convergence angle, optimized drift space, beam spot size at beam waist, and the beam size at solenoid.

The optimized average accelerating gradient $\gamma_{L}^{\prime}$ of the linac can be found by equating the beam's size in the waist (4.11c) to the linac's invariant envelope (2.2):

$$
\gamma_{L}^{\prime} \approx \frac{2}{0.43} \sqrt{\frac{I}{3 I_{0} \sigma_{i}^{2} \gamma}} \approx 7.1 \sqrt{\frac{Q[\mathrm{nC}]}{\sigma_{i}^{2}\left[\mathrm{~cm}^{2}\right] \sigma_{z}[\mathrm{~cm}] \gamma}}[\mathrm{MV} / \mathrm{m}]
$$

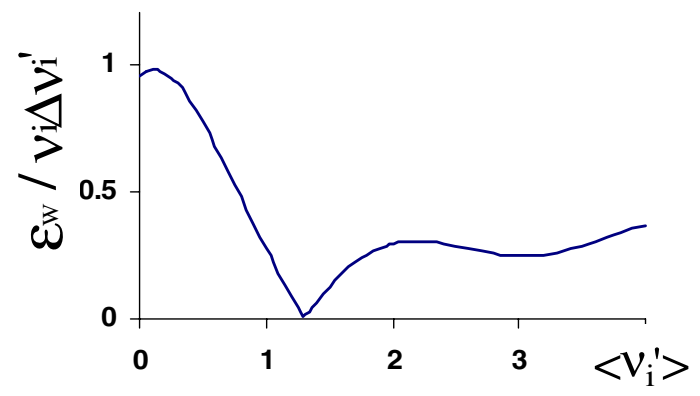

FIG. 4. The relative emittance at the beam's waist $\left(\varepsilon_{W} / \nu_{i} \Delta \nu_{i}^{\prime}\right)$ as a function of the initial convergence angle. Because of the approximation of the spread of the waist's position in Eq. (4.8), the emittance becomes zero near $\left|\bar{\nu}_{i}^{\prime}\right|=1.3$. In reality, this would not be the case, but the minimum would be in the same position. 


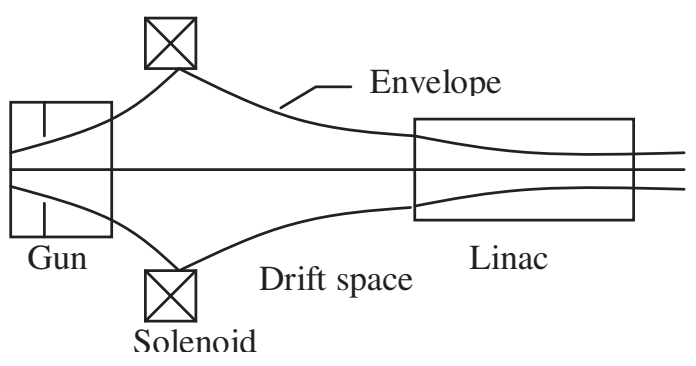

FIG. 5. Scheme of emittance compensation for a compact rf gun + solenoid + drift space + linac system.

\section{THE ROLE OF DESIGNING THE SUPERCONDUCTING GUN SYSTEM}

Figure 5 depicts an emittance compensation of a superconducting cavity system (ignoring chromaticity effects). The scheme for designing and optimizing the system is as follows:

(i) The cavity is designed to have a cathode recess so that the maximum electric field on cathode is about roughly half of the maximum field on axis.

(ii) The spot size on cathode is roughly determined by S$\mathrm{R}$ formula for the normal conducting cavity.

(iii) The solenoid should be as close to the cathode as technically feasible, so that the beam is focused by the solenoid quickly, and the deviation from the invariant envelope reduced. Once the configuration of the location of the solenoid is determined, the beam conditions at the location of the solenoid can be readily assessed by simulation.

(iv) The solenoid strength is adjusted so that $\bar{\nu}_{i}^{\prime} \approx-1.3$, i.e., $\left|\sigma_{i}^{\prime}\right| \approx 0.0244\left(\sqrt{Q[\mathrm{nC}]} / \sqrt{\sigma_{z}[\mathrm{~cm}] \gamma^{3 / 2}}\right)$ in the configuration space (4.11a). The distance from the solenoid exit to the linac entrance is given by: $Z_{W}[\mathrm{~cm}] \approx 40.6 \frac{\sigma_{i}[\mathrm{~cm}] \gamma^{3 / 2}}{\sqrt{Q[\mathrm{nC}]}}$ (4.11b). The waist spot size is therefore given by $\sigma_{W}=$ $\sigma_{i} e^{-\nu_{i}^{\prime 2} / 2} \approx 0.43 \sigma_{i}(4.11 \mathrm{c})$.

(v) The linac gradient is set according to Eq. (4.12) to

$$
\gamma_{L}^{\prime} \approx \frac{2}{0.43} \sqrt{\frac{I}{3 I_{0} \sigma_{i}^{2} \gamma}} \approx 7.1 \sqrt{\frac{Q[\mathrm{nC}]}{\sigma_{i}^{2}\left[\mathrm{~cm}^{2}\right] \sigma_{z}[\mathrm{~cm}] \gamma}}[\mathrm{MV} / \mathrm{m}]
$$

to match condition (2.2), i.e., the invariant envelope of the linac.

\section{ACCOUNTING FOR CHROMATICITY}

\section{A. Rf effects on the distribution of longitudinal phase-space}

The electron energy at the gun's exit $W_{G}$ as a function of initial phase $\phi_{0}$ can be solved numerically. Figure 6 shows the general characteristics of this function. The crest point $\left(\phi_{M}, W_{\max }\right)$ on the curve corresponds to the initial phase of maximum acceleration. The curve has the following properties:

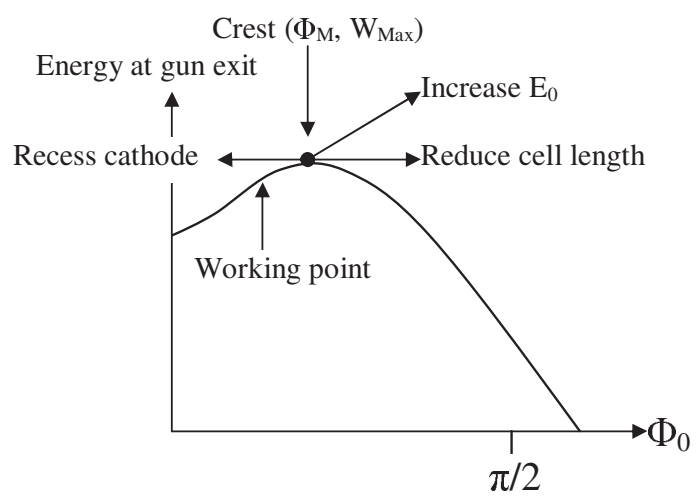

FIG. 6. The energy at gun's exit as a function of the initial phase.

(i) $\phi_{M}$ is always less than $90^{\circ}$. If $\phi_{0}>\phi_{M}$ then $W_{G}$ decreases faster than in the reverse case. $\phi_{0}<\phi_{M}$.

(ii) Increasing the peak field, $E_{0}$ in the gun moves the crest point towards a higher $W_{\max }$ and a higher $\phi_{M}$.

(iii) The recess reduces the field on cathode. This field reduction near cathode increases the phase slippage of the particle due to its nonrelativistic motion, i.e., the particle stays longer time near cathode. Therefore the recess moves the crest point towards a lower value of $\phi_{M}$.

The energy spread introduced by space charge increases the energy of the head of the bunch relative to its tail. Thus, launching a bunch to the left of $\phi_{M}$ minimizes the resulting energy spread. For a high gradient rf gun (such as a pulsed normal-conducting gun) $\phi_{M}$ is relatively high. It is easy to make the launch phase $\phi_{0}$ of the beam smaller than $\phi_{M}$ and so minimize the energy spread. Typically, for such guns, operating at cathode fields of $100 \mathrm{MV} / \mathrm{m}$ or more, and at low charges of about one nanocoulomb, the energy spread is much less than $1 \%$; then, chromaticity can be ignored. However, in a continuous wave (CW) gun, even a superconducting one, the field gradient is limited so that $\phi_{M}$ is low while $\phi_{0}$ must be high to compensate for the lower peak field on the cathode. When these considerations combine with a high bunch charge, then the energy spread of the bunch at the CW gun's exit is considerable. Under these conditions, space-charge forces dominate, and the particles in the front of the bunch have higher energy than those in the rear.

As discussed before, it is desired to have some cathode recess to have better emittance compensation. The cathode recess reduces the field on the cathode and $\phi_{M}$. A shorter cell will compensate this effect.

Figure 7 shows the longitudinal phase space of a 703.5 MHz $1 \frac{1}{2}$ cell superconducting rf gun at its exit. The gun's shape and field distribution are detailed in Sec. VII. For comparison the phase space is shown for an initial phase of $30^{\circ}$, where the energy spread is a minimum (rms of $0.42 \%$ ), and for an initial phase of $60^{\circ}$ ( $\mathrm{rms}$ of $2.86 \%$ ). The charge/bunch is $5 \mathrm{nC}$, the laser on cathode has a transverse uniform distribution with a $5 \mathrm{~mm}$ maximum 


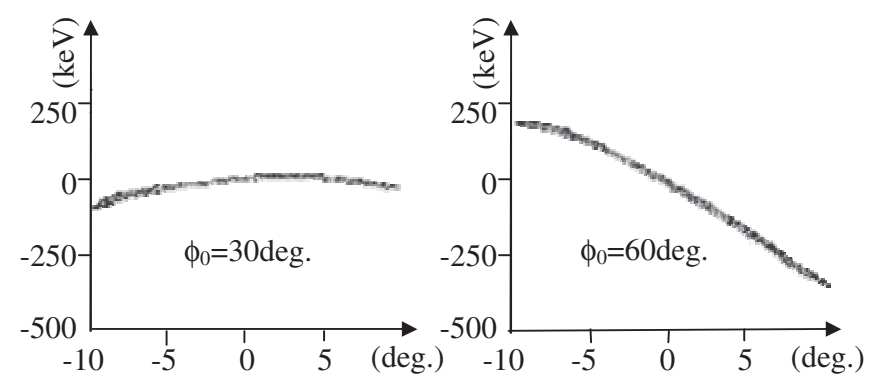

FIG. 7. (Color) Longitudinal phase-space at the gun's exit for $\phi_{0}=30^{\circ}$ (left), and $\phi_{0}=60^{\circ}$ (right).

radius and a longitudinal Gaussian distribution with sigma of 15.8 ps bunch length; the peak field on the axis is $28 \mathrm{MV} / \mathrm{m}$.

\section{B. Rf effects on the distribution of transverse phase-space}

The phase-space angle of a slice $\left(\sigma^{\prime} / \sigma\right)$ at the exit of the gun cavity is given approximately by [3]:

$$
\sigma^{\prime} / \sigma \approx \alpha k \sin \phi
$$

where $\phi$ is the rf phase at the exit of the cavity, $\alpha$ and $k$ are defined in Sec. III. $\phi$ generally is close to $90^{\circ}$ and the bunch's length, $\Delta \phi$, is usually small; therefore, we can ignore the time-dependent $r f$ defocusing. This effect can be seen in the following simulation.

Figure 8 shows the phase spaces of nine slices at the exit of the cavity for the launch phase $\phi_{0}=60^{\circ}$ case. Slice \#1 (front) and slice \#9 (rear) have similar space-charge forces. Despite of their large energy difference, their phase-space angles are quite comparable. The large difference in the phase-space angle between these edge slices (\#s 1,9) and the middle ones (\#s 4, 5, and 6) is due to the difference in space charge. Thus, as we observed above, the slice emittance at this gun's exit is dominated by the dependence of space charge on the slice's position, and not on the timedependent rf force.
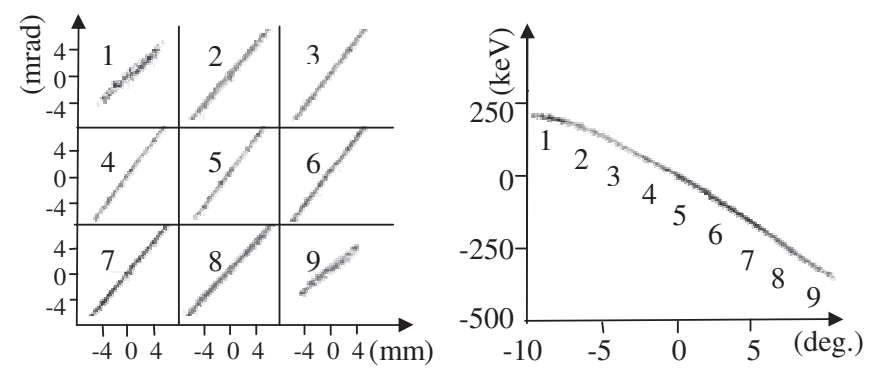

FIG. 8. (Color) Transverse slice phase-spaces (left) and longitudinal slice phase-space (right) at the gun's exit in the example discussed in Sec. VI with $\phi_{0}=60^{\circ}$. Slice \#1 is the front slice and slice \#9 is the rear one.

\section{Chromaticity in the solenoid and drift space}

The solenoid's focusing kick, $\left[F_{S}=(q B / 2 m c \beta \gamma)^{2}\right]$, is proportional to $1 / \gamma^{2}$. As the beam strongly converges at solenoid's exit, the solenoid's contribution to chromaticity is significant as long as the energy spread there is not negligible. Those particles with higher energy (front particles) experience less focusing than those with lower energy (rear particles).

Figure 9 depicts the phase space after the solenoid exit of the previous example; it shows that the solenoid chromaticity cannot be neglected in this case. In fact, the emittance at the solenoid's exit increases from $21.6 \mathrm{~mm}$ mrad to $28.3 \mathrm{~mm}$ mrad in the example due to solenoid chromaticity, while at $\phi_{0}=30^{\circ}$ it only rises from $12.3 \mathrm{~mm}$ mrad to $12.4 \mathrm{~mm}$ mrad.

In the drift space, $\nu(z) \equiv \sigma(z) / \sqrt{P} \propto \gamma^{3 / 2}$, so $\nu_{i}$ is larger for particles of higher energy, and vice versa for those with lower energy.

We note that the space-charge-induced energy spread, acting through chromaticity, adds to the transverse effects of the space charge to enhance the difference between slices, so increasing the spread of the slices' waist positions, and thereby degrading the emittance compensation. To reduce the chromaticity, the initial phase is preferably before the crest point, as shown in Fig. 6. This explains why in previous simulations [7] and experiments [8] the optimized initial phase always was smaller than the theoretical one when the theory ignored chromaticity.

\section{Dealing with the chromaticity of the linac's entrance}

The chromaticity of the focusing of the linac entrance must be considered. The $\mathrm{rf}$ focusing kick is $\theta=\sigma^{\prime} / \sigma=$ $-\gamma_{L}^{\prime} / 2 \gamma$ where $\gamma_{L}^{\prime}$ is the linac's average accelerating gradient. Lower energy particles experience a larger kick angle than those of higher energy. The matching condition for a linac is that the beam must be parallel at the linac's entrance. To compensate for this chromaticity, we can require that the medium energy particles are parallel, with the lower energy particles having a divergence angle (overfocused) and the higher energy ones having a convergence angle (less focused) just before the linac's en-
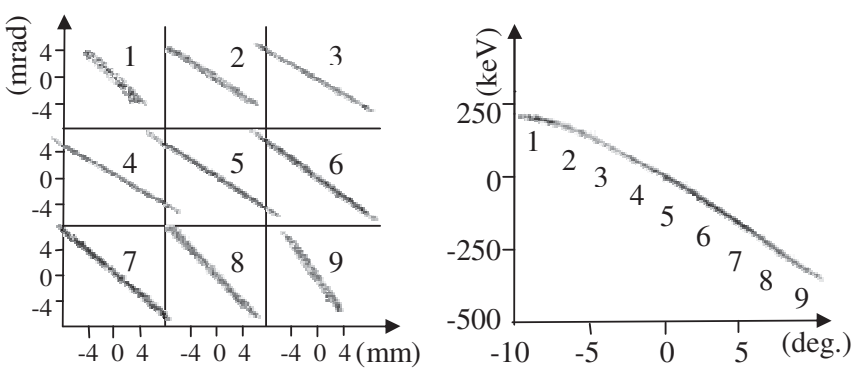

FIG. 9. (Color) Transverse slice phase-spaces (left) and longitudinal slice phase-space (right) at the solenoid's exit in the example discussed in Sec. VI with $\phi_{0}=60^{\circ}$. 


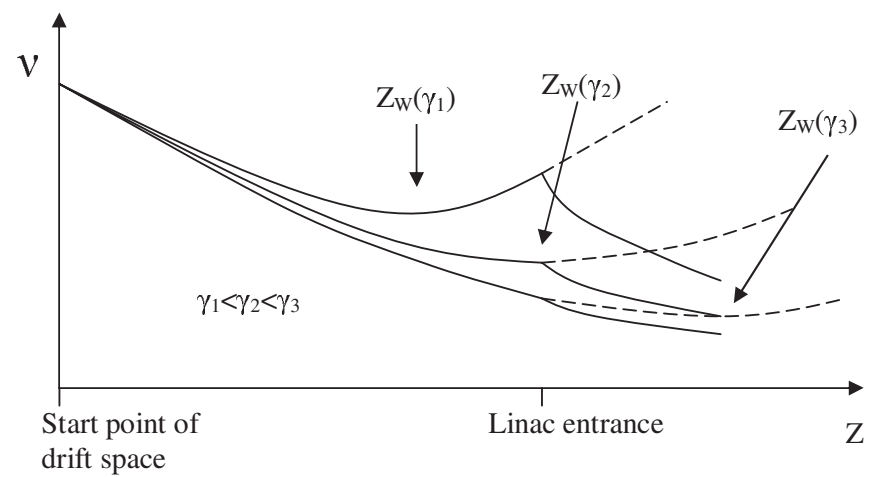

FIG. 10. The chromaticity in drift space. The waist position of the smaller energy slice is earlier and its beam is larger. The waist position of the higher energy slice is later, and its beam is smaller.

trance (Fig. 10). This approach will ensure that the waist of the beam's entire envelope, which is determined by the location of the earliest beam waist, is in front of the linac's entrance.

Figure 11 shows the phase space just before and after the linac's entrance.

To estimate the chromaticity $\Delta \nu_{W D}^{\prime}$ induced in the drift space, we use the simplification that the slices have the same $\nu_{i}$ and the same $\nu_{i}^{\prime}$. From Eq. (4.9) we get

$$
\Delta \nu_{W D}^{\prime} \approx \frac{2 \Delta z_{W}}{\bar{\nu}_{W}} \approx \frac{2 g\left(\nu^{\prime}\right)}{\bar{\nu}_{W}} \frac{d \nu_{i}(\gamma)}{d \gamma} \Delta \gamma=\frac{5.3 \Delta \gamma}{\gamma} .
$$

The linac's entrance gives the variation $\Delta \nu_{W L}^{\prime}$ caused by chromaticity:

$$
\Delta \nu_{W L}^{\prime} \approx \frac{d\left(-\gamma_{L}^{\prime} / 2 \gamma\right)}{d \gamma} \Delta \gamma \bar{\nu}_{W}=\frac{\gamma_{L}^{\prime}}{2 \gamma} \frac{\Delta \gamma}{\gamma} \bar{\nu}_{W} .
$$

$\Delta \nu_{W D}^{\prime}$ should be equal to $\Delta \nu_{W L}^{\prime}$ to minimize the chromatic effect. Therefore,

$$
\gamma_{L}^{\prime}=\frac{10.6 \gamma}{\bar{\nu}_{W}} \approx \frac{10.6 \gamma}{0.43 \nu_{i}}=\frac{10.6}{0.43} \sqrt{\frac{I}{2 I_{0} \sigma_{i}^{2} \gamma}}=46.3 \sqrt{\frac{Q}{\sigma_{i}^{2} \sigma_{z} \gamma}},
$$

where $Q$ is the charge of the bunch in $\mathrm{nC}$, and $\sigma_{i}$ and $\sigma_{z}$ are the beam size and bunch length in $\mathrm{cm}$, respectively.
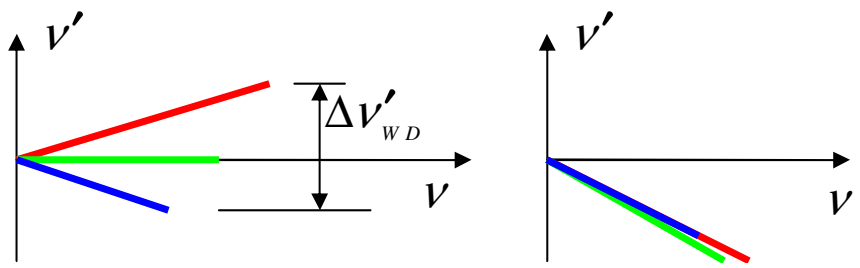

FIG. 11. (Color) The phase-spaces just before (left) and after (right) the linac's entrance. The red, green, and blue lines represent the phase-spaces of the smaller energy, median energy, and higher energy slices, respectively.
Equations (4.12) and (6.4) cannot be met simultaneously. The applied solution must be a compromise. If the energy spread is small, using Eq. (4.12) will give a good result. If the energy spread is large, matching the chromaticity becomes more important, the acceleration is increased or a solenoid is added near the linac entrance to match the chromaticity condition, the beam will have a convergence angle larger than $-\gamma_{L}^{\prime} / 2 \gamma$ required by the invariantenvelope matching condition. Fortunately the effect of the space-charge forces diminishes quadratically as the beam energy increases. Also one can place the linac entrance past the beam waist so that the beam is diverging to reduce the angle mismatch of the invariant envelope. There is no analytical solution and the parameters may be found by using computer optimization techniques. This paper provides a start point for such optimization.

\section{SIMULATION BASED ON THE RHIC ELECTRON-COOLING PROJECT}

Figure 12 shows the configuration that the RHIC cooler will employ.

The gun is expected to be a $1 \frac{1}{2}$ cell $703.5 \mathrm{MHz}$ superconducting rf cavity working in the $\mathrm{CW}$ mode. The charge per bunch in this example is $5 \mathrm{nC}$, one of the options of the RHIC electron-cooling project [9].

The gun cavity's shape and its field lines are shown in Fig. 13.

The cathode is recessed to have rf focusing nearby, and, therefore, the peak field on cathode $E_{c}$ is decreased (Fig. 14). The full cell length (in Fig. 13) is less than

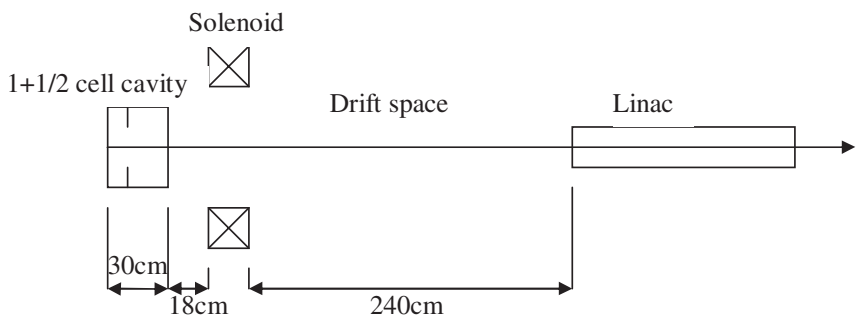

FIG. 12. Schematic layout of the RHIC electron-cooler injector.

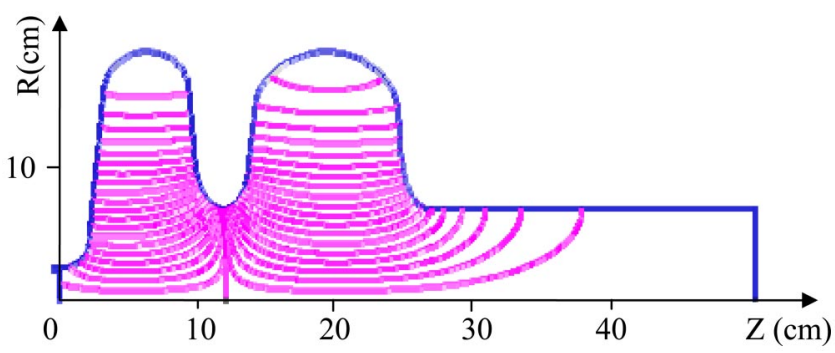

FIG. 13. $1 \frac{1}{2}$ cell rf gun cavity, calculated with SUPERFISH. 


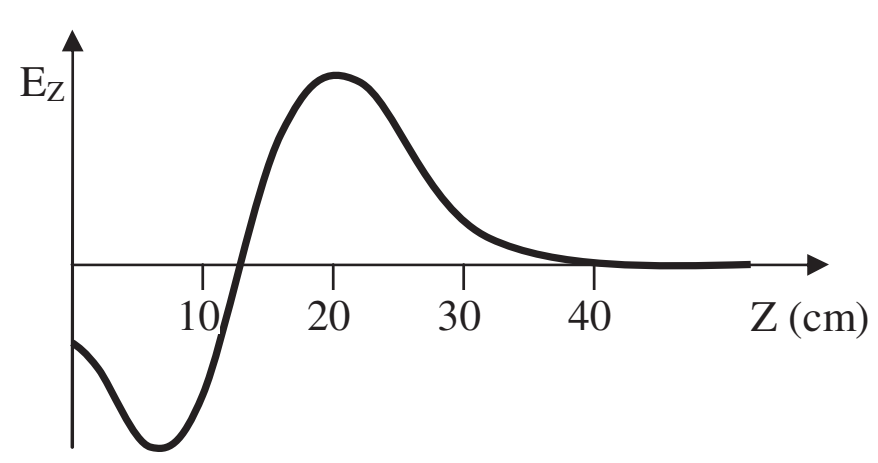

FIG. 14. Field strength on the axis of the rf gun.

$\lambda_{\mathrm{rf}} / 2 \approx 21 \mathrm{~cm}$ to increase $\phi_{M}$ and reduce the energy spread at the gun's exit.

We used the PARMELA code [10] to simulate the beam's dynamics. The beam's energy at gun exit as a function of initial phase is shown in Fig. 15.

The linac uses 5-cell superconducting cavities with the same frequency of gun cavities. The laser distribution is assumed to be uniform transversely, and Gaussian in the longitudinal direction. The longitudinal laser pulse-length is assumed to be $\sigma_{L}[\mathrm{deg}]=4^{\circ}$.

Table I lists the parameters predicted by theory and the results of optimization.

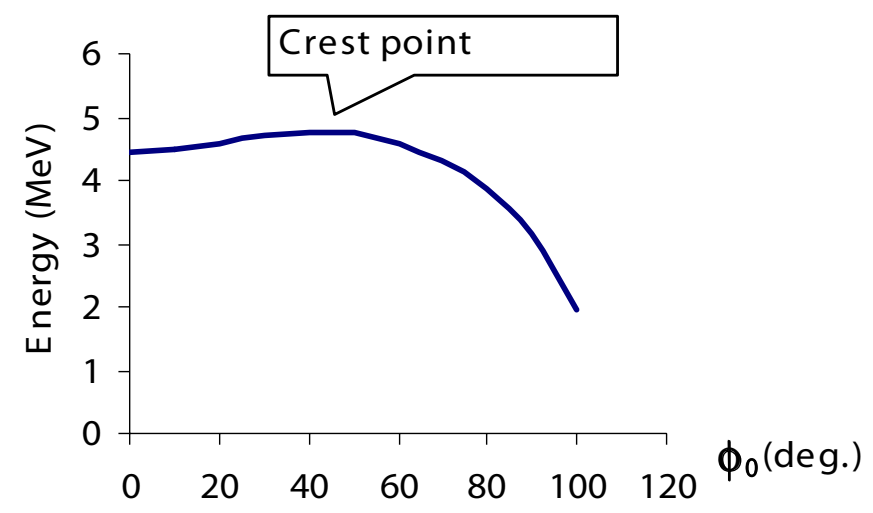

FIG. 15. Electron energy as a function of initial phase $\phi_{0}$.
We assumed in Table I that $\gamma^{\prime}=30 \mathrm{~m}^{-1}\left(E_{0} \approx\right.$ $30 \mathrm{MV} / \mathrm{m}$ ) is the accelerating gradient in the gun and the booster linac. Simulations gave us the rms spot size at the solenoid exit of $\sigma_{i}=0.45 \mathrm{~cm}$, beam energy of about $4.75 \mathrm{MeV}(\gamma=10.3)$, and bunch length $\sigma_{z}=0.55 \mathrm{~cm}$.

The parameters predicted by the theory are close to the results of the optimization, except for the spot size at the linac entrance. This is due to the chromaticity (the rms energy spread is $\sim 0.52 \%$ at linac's entrance). Figure 16 shows the envelope as a function of position for the optimized case. The linac entrance is located behind the beam waist.

Figure 17 shows the emittance as a function of path length. Near the beam waist $(Z \approx 2.4 \mathrm{~m})$ the emittance has a maximum, with minimum at $Z \approx 2 \mathrm{~m}$ and at $Z \approx$ $3 \mathrm{~m}$. This effect is common and shown in simulations in other papers. It is caused by the difference of the phase advance for different slices, which is inversely proportional to the square of the slice radius. Just before the beam waist, the slices reaching their waist earlier rotate faster in phase space than those reaching their waist later. As a result, the slope difference increases. After the beam waist the slices that arrive at their waist later rotate faster and, as a result, the slope difference decreases and the emittance become smaller again. As pointed out in the end of Sec. VI, the linac entrance is located after the beam waist position, where the emittance starts go down. After the linac en-

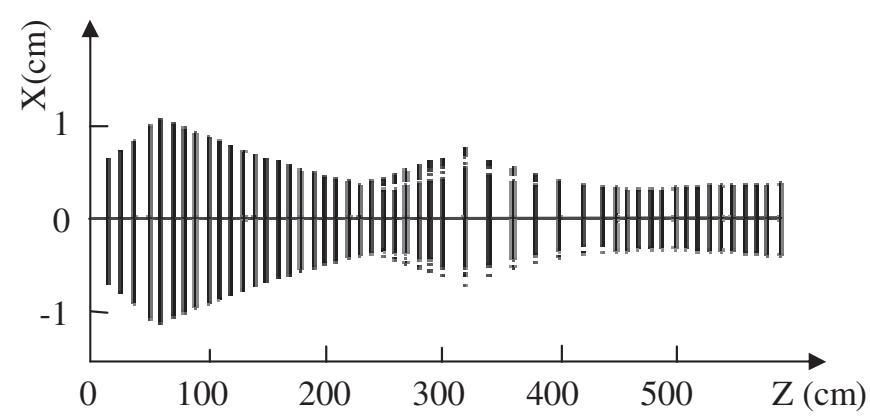

FIG. 16. Beam envelope vs $z$ of an optimized nonmagnetized beam.

TABLE I. Parameters predicted by theory and the results of optimization.

\begin{tabular}{lccc}
\hline \hline & \multicolumn{1}{c}{ Equation } & $\begin{array}{c}\text { Theoretical } \\
\text { predictions }\end{array}$ & $\begin{array}{c}\text { Optimization } \\
\text { results }\end{array}$ \\
\hline Initial phase & $\begin{array}{c}\text { The phase with the minimum } \\
\text { energy spread at the gun's exit }\end{array}$ & From simulation: $\sim 30^{\circ}$ & $30^{\circ}$ \\
Spot size on the cathode & $\sigma_{c}=\sqrt[3]{(c / 2 \pi) Q A / \Lambda \gamma^{1 / 2}}$ & $0.19 \mathrm{~cm}$ & $0.25 \mathrm{~cm}$ \\
Drift space length & $Z_{W}[\mathrm{~cm}] \approx 40.6 \frac{\sigma_{i}[\mathrm{~cm}] \sqrt{\sigma_{z}[\mathrm{~cm}] \gamma^{3 / 2}}}{\sqrt{Q[\mathrm{nC}]}}$ & $2 \mathrm{~m}$ & $2.4 \mathrm{~m}$ \\
Beam size at waist & $\sigma_{W} \approx 0.43 \sigma_{i}$ & $0.19 \mathrm{~cm}$ & $0.13 \mathrm{~cm}$ \\
Matched linac field-gradient without chromaticity & $\gamma_{L}^{\prime} \approx 7.2 \sqrt{\frac{Q[\mathrm{nC}]}{\sigma_{i}^{2}[\mathrm{~cm}] \sigma_{z}[\mathrm{~cm}] \gamma}}[\mathrm{MV} / \mathrm{m}]$ & $15 \mathrm{MV} / \mathrm{m}$ & $30 \mathrm{MV} / \mathrm{m}$ \\
Matched linac field-gradient of chromaticity & $\gamma_{L}^{\prime} \approx 46.3 \sqrt{\frac{Q[\mathrm{nC}]}{\sigma_{i}^{2}\left[\mathrm{~cm}^{2}\right] \sigma_{z}[\mathrm{~cm}] \gamma}}[\mathrm{MV} / \mathrm{m}]$ & $97 \mathrm{MV} / \mathrm{m}$ & \\
\hline \hline
\end{tabular}




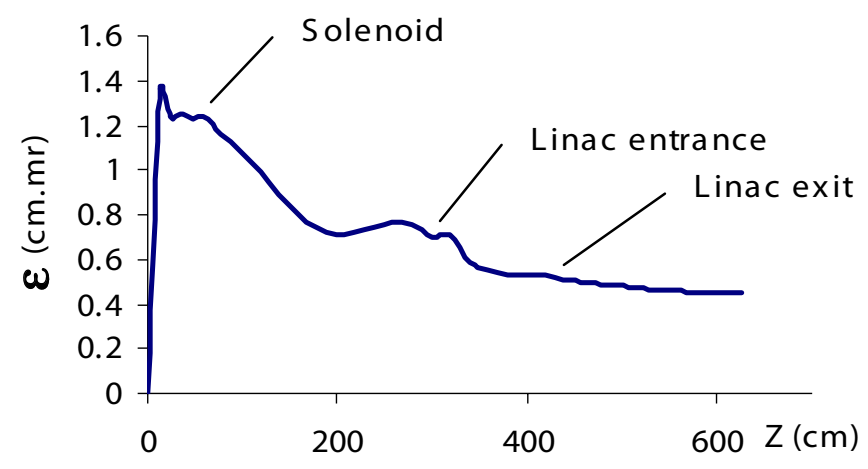

FIG. 17. Emittance $(\varepsilon)$ vs $z$ of the optimized nonmagnetized beam.

trance $(Z \approx 3 \mathrm{~m})$, the chromaticity is compensated. Together with the emittance damping due to the acceleration, the emittance reaches its second minimum at $Z \approx$ $6 \mathrm{~m}$ in this simulation. The final emittance is $4.5 \mathrm{~mm} \mathrm{mrad}$ in this example.

\section{SUMMARY AND CONCLUSION}

We investigated emittance compensation for a compact superconducting rf gun with a solenoid, a drift space, and a linac system. We conclude that it is desirable to recess the cathode and to site the solenoid close to the cathode to approach the invariant envelope of the gun's cavity. The length of the drift space and the spot size at the beam's waist are determined by Eqs. (4.11a)-(4.11c). The linac field gradient is determined by Eq. (4.12). In principle, both of these parameters, along with the solenoid strength, and the linac's accelerating gradient can be determined by the beam's spot size at the solenoid which is easy to obtain by simulation.

The effect of chromaticity generally starts at the solenoid and can be ignored in the gun. The energy spread extends the spread of the slices' waist positions, thereby degrading emittance compensation. A gun cavity should be designed to minimize energy spread at its exit; this can be achieved by shortening the effective length of the cavity cell. The linac gradient required to minimize the chromaticity by focusing at the linac entrance [Eq. (6.4)] is larger than that required for matching the invariant envelope. The applied solution must be a compromise between the two. For a beam with nonzero energy spread, the waist needs to be in front of the linac's entrance.

\section{ACKNOWLEDGMENTS}

The authors wish to thank J. Rosenzweig for useful discussions. Financial support came from the U.S. Department of Energy and from the U.S. Navy.

[1] L. Serafini and J. B. Rosenzweig, Phys. Rev. E 55, 7565 (1997).

[2] S. C. Hartman and J. B. Rosenzweig, Phys. Rev. E 47, 2031 (1993).

[3] K-J Kim, Nucl. Instrum. Methods Phys. Res., Sect. A 275, 201 (1989).

[4] D. Janssen and V. Volkov, Nucl. Instrum. Methods Phys. Res., Sect. A 452, 34 (2000).

[5] J. W. Lewellen and J. Noonan, Phys. Rev. ST Accel. Beams 8, 033502 (2005).

[6] M. Reiser, Theory and Design of Charged Particle Beams (John Wiley \& Sons, New York, 1994), p. 201.

[7] X. Y. Chang, X. J. Wang, and I. Ben-Zvi, in Proceedings of the Particle Accelerator Conference, Chicago, IL, 2001 (IEEE, Piscataway, NJ, 2001).

[8] D. T. Palmer et al., in Proceedings of the Particle Accelerator Conference, Vancouver, BC, Canada, 1997 (IEEE, Piscataway, NJ, 1997).

[9] V. Parkhomchuk and I. Ben-Zvi, Brookhaven National Laboratory Report No. C-A/AP/47, 2001.

[10] L. M. Young, Los Alamos National Laboratory Report No. LA-UR-96-1835 (revised 2001). 